\section{POLLUTION}

Water and Industry

from a Correspondent

IN his introduction to the discussion on freshwater and estuarine effects of industry at the Royal Society on June 3 and 4, Sir Frederick Russell (Marine Biological Association, Plymouth) characterized the state of British rivers and estuaries as the combined result of overpopulation and misplaced priorities. Although the point was not directly taken up in the formal contributions, the effects of further population growth were clearly in the minds of several speakers.

Mr J. H. N. Garland (Water Pollution Research Laboratory, Stevenage) suggested that run-off from agricultural land added to the inorganic nitrogen content of the River Trent only during high flows; the steadier lower levels represent very largely the human contribution. On this basis, he calculated that its pollution load will be half as large again in the 1990s after the anticipated population growth of 1 per cent per annum; at an annual increase of 3 per cent, the Trent at Nottingham would be as filthy as its notorious tributary the Tame is now. This assumes no increase in the agricultural contribution although, in his paper on eutrophication, Dr J. W. G. Lund (Freshwater Biological Association, Ambleside) pointed out that British farmers could profitably use three times as much nitrogen as they do at present. It seems, nevertheless, that the river will be needed as a source of drinking water within 25 years. Mr T. E. Langford (CEGB Freshwater Biology Unit, Ratcliffe-on-Soar) showed that although Drakelow Power Station at Burton discharges cooling water $10^{\circ} \mathrm{C}$ hotter than the river, turbulence caused by pumping adds nearly 4 tonnes of dissolved oxygen per day. It was later agreed that 2-3 tonnes per day of the inorganic nitrogen are nitrified in the cooling towers of this station, which thus makes a positive contribution to the health of the Trent. Some polluting additions are less susceptible to amelioration or control-for example, the high concentrations of zinc, copper and nickel which appear in the Tame after heavy storms wash them from the rooftops of Birmingham.

Mr A. V. Holden (DAFS Freshwater Fisheries Laboratory, Pitlochry) listed sixty different pesticides which may get into freshwater in Britain, showing that although they never reach concentrations lethal to fish, levels at which the hatching of fish eggs is inhibited may be attained in otherwise clean rivers. In Loch Leven, a discharge of dieldrin (which, fortunately, has long since ceased) was accumulated in fish muscle to the extent that this became unfit for human consumption. Egg laying by fish may also be prevented by copper and zinc at well below lethal levels, according to a survey by $\mathrm{Mr} \mathrm{R}$. Lloyd (MAFF Salmon and Freshwater Fisheries Laboratory, London) of problems in determining criteria for water quality. It seemed to be generally agreed by the meeting that such criteria, based on acute toxicity tests against a single species of fish, were not good enough. Mr Lloyd has found that ammonia causes an increase in the rate of urine flow in trout once it exceeded 12 per cent of the lethal concentration, and he has established that fishing conditions in various reaches of the Trent indicate a limit at about this level; it may thus form the basis of a non-lethal bioassay, but some speakers also stressed the need of criteria for invertebrates. It emerged in discussion that some rivers in the Clyde catchment remain fishless, although their waters are now adequately clean. This may be attributable to a poor invertebrate fauna, perhaps because toxic materials are still held in bottom deposits.

Professor J. E. G. Raymont (University of Southampton) opened the proceedings of the second day with a review of the condition of Southampton Water, which showed that it is not suffering too badly, although biological as well as purely planning considerations ought to be taken into account before any further urban development is permitted. An interesting feature is the large American clam Mercenaria mercenaria, the status of which has been elevated from that of a rare immigrant in 1957 to a dominant bivalve which, especially in areas warmed by effluent from Marchwood power station, supports a commercial fishery. Possible problems arise from the ability of these and other filterfeeders to accumulate toxic pollutants even from very low concentrations in the water or sediments.

Dr A. Nelson-Smith (University College, Swansea) dealt with the sources, effects and treatment of oil pollution in estuaries. He concerned himself chiefly with investigations in Milford Haven, but also referred to a saltmarsh in Southampton Water where similar low concentrations of oil in large volumes of refinery effluent have accumulated to the point of denuding 90 acres of both vegetation and several inches of loose sediment. Shores which are reasonably rich in marine life can recover from a single oiling but, within oil ports, pollution is more likely to be of this damaging chronic or repetitive kind. Another rather specialized form of pollution was discussed by $\mathrm{Dr} \mathrm{T}$. H. Pearson (Scottish Marine Biological Association, Oban) who has studied the effects of wastes from a paper mill at the head of a sea loch. Because the sea loch already has local deposits of natural cellulose waste in the form of leaf litter, the fauna is preadapted to deal with pulp wastes, and has merely spread more widely.

\title{
Laser Raman Spectroscopy
}

DURING the past decade light scattering research has been revolutionized by the introduction of the laser. The most widely investigated and useful form of light scattering is Raman scattering, which involves the inelastic scattering of light in media. So far most of the work has focused on the formation of vibrational modes of excitation, but the technique is not confined to this and other forms of excitation are now being studied, for example, an investigation of the pure rotational spectra of gases can provide information on the dimensions of simple molecules. Scattering from magnons or spin waves has furthered understanding of antiferromagnetic materials and their phase transitions. Light beams may also be scattered from plasmas and studies have been made on both gaseous and semiconductor plasmas.

With the high power now available from lasers interpretable Raman spectra can be obtained from dilute systems of molecules or molecular ions in liquid or solid solution. An excellent example of a matrix isolation study using Raman spectroscopy is reported by Ozin and co-workers in next Monday's Nature Physical Science. They have obtained Raman spectra from $\mathrm{SeO}_{2}$ molecules isolated in a solid carbon dioxide matrix at $4.2 \mathrm{~K}$. By measuring spectra at various higher temperatures they were able to observe the results of diffusion controlled chemical reactions including the formation of the dimer $\left(\mathrm{SeO}_{2}\right)_{2}$ and higher polymers.

The matrix isolation technique using molecular crystals was originally developed to deal with problems of molecular structure using infrared spectroscopy. Notable success has been achieved in the area of free radical spectroscopy and the sole evidence for the existence of numerous fragments testifies to the effectiveness of the method. The combination of infrared and laser Raman spectroscopy considerably aids the assignment of the symmetry of molecular species. It is clear that a profitable field of study will be opened up as more workers master the problem of good sample preparation which is so essential for successful Raman spectroscopy. 